\title{
Effects of Increasing Doses of Condensed Tannins Extract from Cistus ladanifer L. on In Vitro Ruminal Fermentation and Biohydrogenation
}

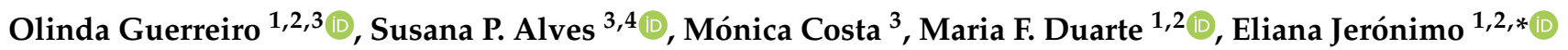 \\ and Rui J. B. Bessa ${ }^{3,4}$ (D) \\ 1 Centro de Biotecnologia Agrícola e Agro-Alimentar do Alentejo (CEBAL), Instituto Politécnico de \\ Beja (IPBeja), 7801-908 Beja, Portugal; olinda.guerreiro@cebal.pt (O.G.); fatima.duarte@cebal.pt (M.F.D.) \\ 2 MED-Mediterranean Institute for Agriculture, Environment and Development, CEBAL, 7801-908 \\ Beja, Portugal \\ 3 Centro de Investigação Interdisciplinar em Sanidade Animal (CIISA), Avenida da Universidade Técnica, \\ 1300-477 Lisboa, Portugal; susanaalves@fmv.ulisboa.pt (S.P.A.); admonicacosta@fmv.ulisboa.pt (M.C.); \\ rjbbessa@fmv.ulisboa.pt (R.J.B.B.) \\ 4 Faculdade de Medicina Veterinária, Universidade de Lisboa, Avenida da Universidade Técnica, \\ 1300-477 Lisboa, Portugal \\ * Correspondence: eliana.jeronimo@cebal.pt
}

check for updates

Citation: Guerreiro, O.; Alves, S.P.; Costa, M.; Duarte, M.F.; Jerónimo, E.; Bessa, R.J.B. Effects of Increasing Doses of Condensed Tannins Extract from Cistus Ladanifer L. on In Vitro Ruminal Fermentation and Biohydrogenation. Animals 2021, 11, 761. https://doi.org/10.3390/ ani11030761

Academic Editor: Gonzalo Hervás

Received: 27 January 2021

Accepted: 8 March 2021

Published: 10 March 2021

Publisher's Note: MDPI stays neutral with regard to jurisdictional claims in published maps and institutional affiliations.

Copyright: (c) 2021 by the authors. Licensee MDPI, Basel, Switzerland. This article is an open access article distributed under the terms and conditions of the Creative Commons Attribution (CC BY) license (https:/ / creativecommons.org/licenses/by/ $4.0 /)$.
Simple Summary: Ruminant edible products have been associated with adverse health effects, due to their high saturated fatty acids and low polyunsaturated fatty acids content, resulting from the extensive biohydrogenation conducted by rumen microbiota. Cistus ladanifer condensed tannins were able to change the lamb ruminal biohydrogenation, increasing the beneficial fatty acids production. The aim of this study was to test the effect of increasing doses of $C$. ladanifer condensed tannins extract $(0,25,50,75$ and $100 \mathrm{~g} / \mathrm{kg}$ dry matter $)$ on in vitro rumen fermentation and biohydrogenation. The increasing doses of condensed tannins led to a moderate decrease of volatile fatty acids production, a pronounced depression in microbial odd and branched fatty acids and of dimethyl acetals production, and a minor effect on the biohydrogenation, which indicates that microbial growth was more inhibited than fermentative and biohydrogenation activities. The ability of $C$. ladanifer condensed tannins extract to modulate the biohydrogenation $(\mathrm{BH})$ was not observed in the present study. However, the results obtained suggest a possible adaptative response of the microbial population to stress stimuli of condensed tannins and lipid supplementation.

Abstract: Cistus ladanifer (rockrose) is a perennial shrub quite abundant in the Mediterranean region, and it is a rich source in secondary compounds such as condensed tannins (CTs). Condensed tannins from $C$. ladanifer were able to change the ruminal biohydrogenation $(\mathrm{BH})$, increasing the $t 11-18: 1$ and $c 9, t 11-18: 2$ production. However, the adequate conditions of the C. ladanifer CTs used to optimize the production of $t 11-18: 1$ and $c 9, t 11-18: 2$ is not yet known. Thus, we tested the effect of increasing the doses of C. ladanifer CT extract $(0,25,50,75$ and $100 \mathrm{~g} / \mathrm{kg}$ dry matter (DM)) on in vitro rumen $\mathrm{BH}$. Five in vitro batch incubations replicates were conducted using an oil supplemented high-concentrate substrate, incubated for $24 \mathrm{~h}$ with $6 \mathrm{~mL}$ of buffered ruminal fluid. Volatile fatty acids (VFAs) and long chain fatty acids (FA) were analyzed at $0 \mathrm{~h}$ and $24 \mathrm{~h}$, and $\mathrm{BH}$ of $c 9-18: 1, c 9, c 12-18: 2$ and $c 9, c 12$, $c 15-18: 3$, and BH products yield were computed. Increasing doses of $C$. ladanifer CTs led to a moderate linear decrease $(p<0.001)$ of the VFA production (a reduction of $27 \%$ with the highest dose compared to control). The disappearance of $c 9-18: 1$ and $c 9, c 12-18: 2$ as well as the production of $t 11-18: 1$ and $c 9$, $t$ 11:18:2 was not affected by increasing doses of $C$. ladanifer $\mathrm{CTs}$, and only the disappearance of $c 9$, $c 12, c 15-18: 3$ suffered a mild linear decrease (a reduction of $24 \%$ with the highest dose compared to control). Nevertheless, increasing the $C$. ladanifer CT dose led to a strong depression of microbial odd and branched fatty acids and of dimethyl acetals production (less than 65\% with the highest dose compared to control), which indicates that microbial growth was more inhibited than fermentative and biohydrogenation activities, in a possible adaptative response of microbial population to stress induced to CTs and polyunsaturated fatty acids. The ability of $C$. ladanifer to modulate the ruminal 
$\mathrm{BH}$ was not verified in the current in vitro experimental conditions, emphasizing the inconsistent $\mathrm{BH}$ response to CTs and highlighting the need to continue seeking the optimal conditions for using CTs to improve the fatty acid profile of ruminant fat.

Keywords: Mediterranean shrub; phenolic compound; rumen metabolism; fatty acids; biohydrogenation intermediates; dimethyl acetals

\section{Introduction}

Tannins are a group of plant secondary compounds, defined as naturally occurring water-soluble polyphenols that have the ability to precipitate proteins $[1,2]$. Tannins are generally classified as hydrolysable and condensed tannins (CTs). Condensed tannin polymers vary tremendously in their constituent monomers, stereochemistry, polymer size, and intermolecular linkages, in addition to their concentration [3] and are generally considered as antinutritional factors due to their adverse effects on feed intake, nutrient utilization and toxicity [4]. However, CTs have also been recognized as useful phytochemicals for modulating rumen microbial fermentation [2], and ruminal biohydrogenation (BH) of dietary unsaturated fatty acids (FAs) [5-7].

Ruminant edible products have been associated with adverse health effects, due to their high saturated FA (SFA) and low polyunsaturated FA (PUFA) content, due to the extensive $\mathrm{BH}$ conducted by rumen microbiota [8]. Therefore, manipulation of the ruminal $\mathrm{BH}$ has been attempted as way if improving the nutritional value of ruminant fat, by increasing the rumen outflow of the dietary PUFA and the beneficial biohydrogenation intermediates, such as vaccenic acid (t11-18:1) and rumenic acid $(c 9, t 11-18: 2)[9,10]$. Some in vitro studies have suggested that CTs may be efficient in inhibiting the last step of $\mathrm{BH}$, decreasing the 18:0 formation and leading to t11-18:1 accumulation [5,6,11]. However, other studies reported a general inhibition or stimulation of $\mathrm{BH}$ without the inhibition of its last step $[7,12,13]$.

Cistus ladanifer L. is a shrub widely spread over the Mediterranean region and often used by grazing ruminants, particularly during periods of pasture scarcity [14]. The inclusion of the $C$. ladanifer plant into oil-supplemented diets modified the lamb abomasal and meat FA composition [15-17]. When included in an oil-supplemented high-forage diet, $C$. ladanifer induced a large increase in t11-18:1 in abomasal digesta and increased the deposition of $t 11-18: 1$ and $c 9, t 11-18: 2$ in the intramuscular fat of lambs [15]. An increase in the trans-18:1 isomers in digestive contents (rumen and abomasum) and the intramuscular fat of lambs was also obtained with the inclusion of the $C$. ladanifer plant in oil-supplemented medium-forage diet $[17,18]$. Cistus ladanifer presents high amounts of CTs $(40-160 \mathrm{~g} / \mathrm{kg}$ dry matter (DM)) [14], and its effects on ruminal BH seems to be associated with action of CTs on ruminal metabolism. Comparing the effect of several secondary compound fractions present in C. ladanifer (essential oil, dichloromethane extract, total phenolics, non-tannin phenols and condensed tannins fractions) on in vitro rumen $\mathrm{BH}$, was possible to observe that $\mathrm{CT}$ extract is the secondary compound fraction of $C$. ladanifer, with highest capacity to modulate the $\mathrm{BH}$ pattern, inducing a larger accumulation of t11-18:1 [7].

Such results showed that $C$. ladanifer $\mathrm{CTs}$ can modulate the ruminal $\mathrm{BH}$ and potentially improve the nutritional value of ruminant fat. However, as observed for other CT sources, the effects of CTs from C. ladanifer on BH might change with the dose [11,13]. Moreover, there is increasing evidence that the effect of tannins on ruminal $\mathrm{BH}$ depends on the features of the basal diet [19]. The ability of $C$. ladanifer $C T$ extract to modulate the in vitro ruminal $\mathrm{BH}$ was only demonstrated using an oil-supplemented high-forage substrate $[7,12]$. Thus, the present in vitro experiment was designed to evaluate the effect of increasing doses of CT extract from C. ladanifer on rumen fermentation and $\mathrm{BH}$ when an oil-supplemented high-concentrate substrate is used. Moreover, the present study also aimed to determine the dose of C. ladanifer CT doses which optimize the $c 9, t 11-18: 2$ and $t 11: 18: 1$ production. 


\section{Materials and Methods}

\subsection{Cistus Ladanifer Sampling}

Cistus ladanifer aerial parts were harvested in January 2013, in the Baixo Alentejo region, in Monte do Vento, Mértola, southern Portugal $\left(37^{\circ} 48^{\prime} 28.17^{\prime \prime} \mathrm{N}-7^{\circ} 40^{\prime} 39.08^{\prime \prime} \mathrm{W}\right)$, in a parcel of holm oak forest of Quercus rotundifolia L., where naturally occurring C. ladanifer plants are the predominant vegetation. Samples were manually harvested with scissors and kept at $-20{ }^{\circ} \mathrm{C}$ for 1 week until further use. The collected aerial part of plants was composed of leaves and soft stems.

\subsection{Preparation of Cistus Ladanifer Condensed Tannins Extract}

The condensed tannin (CT) extract was obtained by the sequential extraction of C. ladanifer aerial parts and purified using a Sephadex LH-20 chromatographic column (GE Healthcare Bio-Science, Uppsala, Sweden), as described by Guerreiro et al. [7]. Condensed tannin extract was maintained at $-20{ }^{\circ} \mathrm{C}$ until in vitro incubation. Condensed tannin extract presents $600 \mathrm{~g}$ of $\mathrm{CT} / \mathrm{kg}$ DM, determined using C. ladanifer purified $\mathrm{CT}$ as standard and following the methodology described in Guerreiro et al. [14].

\subsection{In Vitro Incubation with Ruminal Fluid}

The experimental animal procedures were approved by the Ethical and Animal WellBeing Commission (CEBEA) of the Faculty of Veterinary Medicine, University of Lisbon, Portugal (Protocol FMV/CEBEA 007/2016). All methods and procedures were performed following the established guidelines from this institution and following compliance guidelines of European Union (Directive 86/609/EEC). Additionally, authors O. Guerreiro, M. Costa, S.P. Alves and R.J.B. Bessa hold a FELASA (Federation of European Laboratory Animal Society Associations) grade $C$ certificate that enables them to design and carry out animal experimentation under the European Union regulations.

Ruminal fluid was collected from two rumen-fistulated Merino Branco rams $(40 \pm 2 \mathrm{~kg}$ live weight), fed daily $600 \mathrm{~g}$ of commercial concentrate and $600 \mathrm{~g}$ of oat hay, in two equal meals at 9:30 and 17:00 h. The concentrate mixture comprised maize, soybean, sunflower, wheat, wheat bran and rape (220 g crude protein; $95 \mathrm{~g}$ crude fiber; $65 \mathrm{~g}$ ash; $35 \mathrm{~g}$ ether extract; 4 g sodium; 7500 IU of vitamin $\mathrm{A} ; 1500 \mathrm{IU}$ of vitamin $\mathrm{D}_{3}$ and $7.5 \mathrm{mg}$ of vitamin E; per $\mathrm{kg}$ of DM). Ruminal fluid was collected just before the morning feeding in warm flasks (approximately $39^{\circ} \mathrm{C}$ ) and strained through four layers of gauze. Strained ruminal fluid from both rams was pooled and it was diluted with a phosphate-bicarbonate buffer solution [20], pre-warmed at $39^{\circ} \mathrm{C}$ and saturated with $\mathrm{CO}_{2}$, in a proportion of 1:4 (ruminal fluid:buffer solution, $v / v$ ), under constant $\mathrm{CO}_{2}$ flux. Before adding the ruminal fluid, a redox indicator (resazurin solution, $0.1 \%(w / v))$ and a reducing agent $(625 \mathrm{mg}$ of L-cysteine- $\mathrm{HCl}$, $1 \mathrm{~N}$ of sodium hydroxide and $625 \mathrm{mg}$ of sodium sulfide nonahydrate) were added to the buffer solution. Reduction was indicated upon a change in color of the blue resazurin to colorless dihydroresorufin.

After the complete reduction of the buffer solution and mixture with strained ruminal fluid, $6 \mathrm{~mL}$ of buffered ruminal fluid was added to Hungate tubes containing $60 \mathrm{mg}$ DM of ground feed substrate (control), or $60 \mathrm{mg}$ DM of feed substrate plus each dose of $C$. ladanifer $\mathrm{CT}$ extract $(1.5,3.0,4.5$ and $6 \mathrm{mg})$. The final concentrations of added C. ladanifer CT extract were $25,50,75$ and $100 \mathrm{~g} / \mathrm{kg} \mathrm{DM}$, corresponding to $1.5 \%, 3 \%, 4.5 \%$ and $6 \%$ of $\mathrm{CT}$, respectively. The basal substrate was composed of a mixture of commercial concentrate (564 g/ $\mathrm{kg} \mathrm{DM})$, grass hay ( $376 \mathrm{~g} / \mathrm{kg} \mathrm{DM})$ and sunflower oil $(60 \mathrm{~g} / \mathrm{kg} \mathrm{DM})$. The commercial concentrate was the same used to feed the rumen content donors. In this work, a highconcentrate diet was used, which is a type of diet typically used in ruminant production systems, particularly in the finishing stage. Dietary supplementation with unsaturated fatty acids has been extensively tested to improve the nutritional value of ruminant fat, and vegetable oil levels up to $6 \%$ have been successfully applied in lamb and in this context without compromising the ruminal metabolism and animal productivity $[15,21-23]$. 
The tubes were then filled with $\mathrm{CO}_{2}$, thus ensuring oxygen-free conditions and closed with a butyl rubber stopper and screw cap. The tubes allocated to the $0 \mathrm{~h}$ incubation time were immediately frozen at $-20{ }^{\circ} \mathrm{C}$ after the addition of the buffered ruminal fluid. Incubation was performed in a water-bath (Unitronic Pro, JP Selecta, Barcelona, Spain) at $39^{\circ} \mathrm{C}$ with gentle agitation for $24 \mathrm{~h}$. At 0 and $24 \mathrm{~h}$ of incubation, the $\mathrm{pH}$ of ruminal fluid was determined. At the end of the incubation, tubes were immediately frozen at $-20{ }^{\circ} \mathrm{C}$. For each treatment and incubation time, 2 Hungate tubes were used, one for volatile fatty acid (VFA) determination and other tube for other FA determination. The allocation of tubes to the treatments, incubation time, order of filling with buffered ruminal fluid, and to the position in the water-bath were randomized. The incubation procedure was replicated 5 times in 5 consecutive weeks.

The incubation tubes used for FA analysis were freeze-dried (ScanVac CoolSafe, LaboGene ApS, Lynge, Denmark), and stored at $-20^{\circ} \mathrm{C}$ until analysis. The tubes for analysis of VFA were kept at $-20^{\circ} \mathrm{C}$ until analysis.

\subsection{Fatty Acids Analysis}

Volatile FAs (VFAs) were analyzed by gas chromatography with flame ionization detection (GC-FID) using a Shimadzu GC-2010 Plus chromatograph (Shimadzu, Kyoto, Japan) equipped with a fused silica capillary column (Nukol, $30 \mathrm{~m}, 0.25 \mathrm{~mm}$ i.d., $0.25 \mu \mathrm{m}$ film thickness, Supelco, Sigma-Aldrich, St. Louis, MO, USA), as described by Oliveira et al. [24].

The contents of each incubation tube was transesterified to prepare FA methyl esters and dimethyl acetals (DMAs) by using a combined basic followed by acid catalysis adapted from Jenkins [25] and modified by Alves et al. [26], used as the internal standard 19:0 ( $1 \mathrm{mg} / \mathrm{mL}$ ). Fatty acid methyl esters were separated by gas chromatography using a Shimadzu GC-2010 Plus chromatograph (Shimadzu, Kyoto, Japan) equipped with a SP2560 capillary column $(100 \mathrm{~m}, 0.25 \mathrm{~mm}$ i.d., $0.20 \mu \mathrm{m}$ film thickness, Supelco, SigmaAldrich Inc., Bellefont, PA, USA). The peak identification was based on the comparison of retention times with FA methyl esters standards (37 component FAME mix and bacterial acid methyl esters mix from Supelco, Sigma-Aldrich Inc., Bellefont, PA, USA) and by comparison with published chromatograms [26]. Identifications were confirmed by gas chromatography mass spectrometry using a Shimadzu GC-MS QP2010-Plus instrument (Shimadzu, Kyoto, Japan).

\subsection{Calculations}

The VFA and FA (except C18 FA) and DMA productions during the incubation time were calculated directly from their concentrations at $24 \mathrm{~h}$ minus $0 \mathrm{~h}$ incubation times (presented in the Supplementary Table S1). The C18 FA differences between $24 \mathrm{~h}$ and $0 \mathrm{~h}$ were computed using the mean content of the total amount of C18 FA in each pair of tubes (presented in the Supplementary Table S2) and the relative distribution of C18 FA (in \% of total C18 FA) present at the tube from $0 \mathrm{~h}$ and $24 \mathrm{~h}$ incubation time as detailed by [7].

The biohydrogenation of dietary unsaturated C18 FA were then calculated as:

$$
B H \text { of } F A(\%)=\frac{[\mathrm{FA} 0 \mathrm{~h}]-[\mathrm{FA} 24 \mathrm{~h}]}{[\mathrm{FA} 0 \mathrm{~h}]} \times 100
$$

where FA can be $c 9-18: 1, c 9, c 12-18: 2$ or $c 9, c 12, c 15-18: 2$ and $\mathrm{BH}$ stands for biohydrogenation.

All C18 FAs that displayed a positive balance during the $24 \mathrm{~h}$ of incubation were considered here as biohydrogenation products (BHPs). The relative yield of the main classes of BHPs (18:0, 18:1 isomers; 18:2 isomers and oxo-FA) were computed from the C18 FA balance data and expressed in percentage of total BHP, as detailed by [7].

The ratios between $\mathrm{BH}$ of each dietary $\mathrm{C} 18$ unsaturated FA $(c 9-18: 1, c 9, c 12-18: 2$ and $c 9, c 12, c 15-18: 3)$ and the total VFA were also computed. 


\subsection{Statistical Analysis}

Data were analyzed as a randomized complete block design using the Proc Mixed of SAS (SAS Institute inc, Cary, NC, USA), considering the tube as the experimental unit, and the "incubation run" as a random block according to the following model:

$$
Y i j=\mu+T i+D j+e i j
$$

where $Y i j$ is the observation, $\mu$ is the overall mean, $T i$ is the fixed effect of treatment (CT doses), $D j$ is the random effect of incubation run and eij is the residual error. Orthogonal contrasts were used to detect linear and quadratic responses. When significant effects of treatments were detected, the least square means were compared using the pairwise Tukey comparison test. Differences were declared significant at $p<0.05$.

\section{Results}

The net production of the total VFA and of the individual VFA are presented in Table 1. Increasing the dose CT caused a linear decrease in the total VFA production $(p<0.001)$ and in all the individual VFA production, except for iso-5:0, that decreased quadratically $(p=0.004)$. However, the 2:0/3:0 ratio was not influenced by CTs, averaging 3.0. Cistus ladanifer CT extract doses did not affect the $\mathrm{pH}$ of fluid ruminal at $24 \mathrm{~h}$ of incubation $(p<0.05$; average of 6.74).

Table 1. Effect of increasing doses of Cistus ladanifer condensed tannin (CT) extract $(0,25,50,75$ and $100 \mathrm{~g} / \mathrm{kg}$ dry matter (DM)) on net production of volatile fatty acid (VFA, mmol/L) during the $24 \mathrm{~h}$ of incubation.

\begin{tabular}{|c|c|c|c|c|c|c|c|c|}
\hline & \multicolumn{5}{|c|}{ C. ladanifer CT, g/kg Dry Matter } & \multirow{2}{*}{ SEM } & \multicolumn{2}{|c|}{$p$ Values } \\
\hline & 0 & 25 & 50 & 75 & 100 & & Linear & Quadratic \\
\hline Total VFA & 35.4 & 33.2 & 32.6 & 26.6 & 25.8 & 1.59 & $<0.001$ & 0.629 \\
\hline $2: 0$ & 22.0 & 20.9 & 20.7 & 16.4 & 16.3 & 1.20 & $<0.001$ & 0.623 \\
\hline $3: 0$ & 7.28 & 6.79 & 6.62 & 5.60 & 5.19 & 0.314 & $<0.001$ & 0.554 \\
\hline iso- $4: 0$ & 0.41 & 0.24 & 0.26 & 0.20 & 0.16 & 0.042 & $<0.001$ & 0.219 \\
\hline $4: 0$ & 4.43 & 4.33 & 4.15 & 3.68 & 3.45 & 0.205 & $<0.001$ & 0.190 \\
\hline iso- $5: 0$ & 0.61 & 0.46 & 0.41 & 0.36 & 0.33 & 0.025 & $<0.001$ & 0.004 \\
\hline $5: 0$ & 0.50 & 0.40 & 0.37 & 0.30 & 0.27 & 0.020 & $<0.001$ & 0.107 \\
\hline $2: 0 / 3: 0$ ratio & 3.04 & 3.09 & 3.02 & 2.92 & 3.13 & 0.138 & 0.734 & 0.738 \\
\hline
\end{tabular}

SEM, standard error of the mean.

The BH for dietary unsaturated C18 FAs are presented in Table 2. The BH of $c 9-18: 1$ and $c 9, c 12-18: 2$ did not differ $(p>0.05)$ among treatments. The BH of $c 9, c 12, c 15-18: 3$ decreased linearly with the increasing doses of CT $(p=0.003)$. The CT had no effect on the yield of 18:0 and sum of 18:1 BHP. The yield of $18: 0$ ranged from 45.6 to $50.2 \%$ of total BHP, and the yield of 18:1 BHP ranged 34.0 to $35.9 \%$ of total BHP. However, the yield of 18:2 BHP decreased linearly with the increasing dose of CT. The yield of oxo-FA showed a quadratic increase $(p=0.001)$ being higher in treatments with CT (average 14.3\%) than in the control (11.5\%). 
Table 2. Effect of increasing doses of Cistus ladanifer condensed tannin (CT) extract $(0,25,50,75$ and $100 \mathrm{~g} / \mathrm{kg} \mathrm{DM}) \mathrm{on}$ dietary $\mathrm{C} 18$ unsaturated fatty acids biohydrogenation $(\mathrm{BH})$, relative yield in the $\mathrm{BH}$ products, and on $\mathrm{BH} /$ volatile fatty acid (VFA) ratio.

\begin{tabular}{|c|c|c|c|c|c|c|c|c|}
\hline & \multicolumn{5}{|c|}{ C. ladanifer $\mathrm{CT}, \mathrm{g} / \mathrm{kg}$ Dry Matter } & \multirow{2}{*}{ SEM } & \multicolumn{2}{|c|}{$p$ Values } \\
\hline & 0 & 25 & 50 & 75 & 100 & & Linear & Quadratic \\
\hline \multicolumn{9}{|c|}{ Biohydrogenation (\%) } \\
\hline c9-18:1 & 51.2 & 52.4 & 47.1 & 48.6 & 48.5 & 4.30 & 0.426 & 0.760 \\
\hline$c 9, c 12-18: 2$ & 68.7 & 67.1 & 63.4 & 64.6 & 65.0 & 3.93 & 0.431 & 0.545 \\
\hline$c 9, c 12, c 15-18: 3$ & 67.6 & 61.2 & 52.3 & 54.7 & 50.8 & 3.76 & 0.003 & 0.239 \\
\hline \multicolumn{9}{|c|}{ Biohydrogenation Products (\%) } \\
\hline 18:0 & 49.2 & 47.8 & 45.6 & 50.2 & 49.5 & 3.50 & 0.666 & 0.327 \\
\hline $18: 1^{1}$ & 35.8 & 35.3 & 35.9 & 34.0 & 35.0 & 4.65 & 0.553 & 0.952 \\
\hline $18: 2^{2}$ & 4.18 & 3.36 & 3.44 & 2.36 & 2.34 & 0.608 & 0.020 & 0.841 \\
\hline oxo-FA $^{3}$ & 11.5 & 14.1 & 15.4 & 13.9 & 13.6 & 2.15 & 0.046 & 0.001 \\
\hline \multicolumn{9}{|c|}{ Biohydrogenation:VFA ratio } \\
\hline $\begin{array}{r}c 9- \\
18: 1 \mathrm{BH} / \mathrm{VFA}\end{array}$ & 1.47 & 1.59 & 1.45 & 1.86 & 1.95 & 0.182 & 0.009 & 0.340 \\
\hline $\begin{array}{r}c 9, c 12- \\
18: 2 \mathrm{BH} / \mathrm{VFA}\end{array}$ & 1.95 & 2.03 & 1.95 & 2.47 & 2.58 & 0.171 & 0.004 & 0.278 \\
\hline $\begin{array}{r}c 9, c 12, c 15- \\
18: 3 \mathrm{BH} / \mathrm{VFA}\end{array}$ & 1.93 & 1.85 & 1.60 & 2.10 & 2.01 & 0.159 & 0.437 & 0.235 \\
\hline
\end{tabular}

SEM, standard error of the mean. ${ }^{1}$ Sum of 18:1 isomers, all 18:1 biohydrogenation intermediates, i.e., all 18:1 FA listed in the Table $3 ;^{2}$ Sum of 18:2 isomers, all 18:2 biohydrogenation intermediates, i.e., all 18:2 FA listed in the Table $3{ }^{3}{ }^{3}$ Sum of 10-oxo-18:0 and 13-oxo-18:0.

Table 3. Effect of increasing doses of Cistus ladanifer condensed tannins (CT) extract (0, 25, 50, 75 and $100 \mathrm{~g} / \mathrm{kg}$ DM) on net C18 fatty acid changes ( $\mu \mathrm{g} /$ tube) during the $24 \mathrm{~h}$ of incubation.

\begin{tabular}{|c|c|c|c|c|c|c|c|c|}
\hline & \multicolumn{5}{|c|}{ C. ladanifer CT, g/kg Dry Matter } & \multirow{2}{*}{ SEM } & \multicolumn{2}{|c|}{$p$ Values } \\
\hline & 0 & 25 & 50 & 75 & 100 & & Linear & Quadratic \\
\hline \multicolumn{9}{|l|}{ C18 FA loss } \\
\hline c9-18:1 & -635 & -646 & -576 & -597 & -597 & 57.5 & 0.479 & 0.730 \\
\hline$c 9, c 12-18: 2$ & -1004 & -946 & -827 & -896 & -956 & 240.2 & 0.617 & 0.230 \\
\hline $\begin{array}{r}c 9, c 12, c 15- \\
18 \cdot 3\end{array}$ & -23.5 & -21.5 & -16.9 & -19.4 & -18.4 & 3.61 & 0.061 & 0.233 \\
\hline $\begin{array}{l}10.5 \\
\text { Sum }\end{array}$ & -1663 & -1613 & -1420 & -1512 & -1572 & 259.4 & 0.544 & 0.364 \\
\hline \multicolumn{9}{|l|}{ C18 FA gains } \\
\hline $18: 0$ & 774 & 750 & 656 & 743 & 753 & 100.1 & 0.836 & 0.380 \\
\hline \multicolumn{9}{|l|}{$18: 1$ isomers } \\
\hline$t 4-$ & 2.84 & 3.33 & 3.60 & 4.44 & 4.68 & 0.718 & $<0.001$ & 0.947 \\
\hline$t 5$ & 1.25 & 0.93 & 2.08 & 2.40 & 3.27 & 0.815 & 0.002 & 0.395 \\
\hline$t 6-, t 7-, t 8-$ & 22.8 & 27.1 & 27.1 & 31.5 & 33.3 & 4.73 & 0.009 & 0.960 \\
\hline t9- & 61.7 & 54.7 & 43.4 & 42.2 & 40.2 & 6.40 & $<0.001$ & 0.538 \\
\hline$t 10-$ & 33.6 & 40.7 & 40.4 & 42.5 & 41.8 & 7.18 & 0.276 & 0.493 \\
\hline$t 11-$ & 456 & 397 & 324 & 333 & 376 & 136.9 & 0.186 & 0.155 \\
\hline$t 12-$ & 27.4 & 29.8 & 28.6 & 32.2 & 33.7 & 4.79 & 0.131 & 0.780 \\
\hline$t 15-$ & 14.3 & 16.4 & 15.1 & 19.0 & 20.7 & 3.08 & 0.025 & 0.566 \\
\hline$c 11-$ & -10.6 & -11.5 & -8.12 & -10.0 & -9.42 & 2.90 & 0.518 & 0.747 \\
\hline$c 12-$ & 22.2 & 21.2 & 18.5 & 20.0 & 21.2 & 4.23 & 0.695 & 0.391 \\
\hline$c 13-$ & 1.16 & 1.05 & 0.35 & 1.75 & 1.34 & 0.289 & 0.268 & 0.180 \\
\hline$t 16-, t 14-$ & 12.5 & 15.8 & 14.3 & 16.9 & 18.3 & 2.75 & 0.028 & 0.951 \\
\hline$c 16-$ & 2.99 & 3.29 & 3.09 & 2.92 & 3.39 & 0.678 & 0.634 & 0.736 \\
\hline Sum & 654 & 607 & 522 & 544 & 593 & 153.2 & 0.371 & 0.227 \\
\hline \multicolumn{9}{|l|}{$18: 2$ isomers } \\
\hline$t 9, t 12-$ & 9.47 & 6.33 & 5.34 & 4.18 & 3.47 & 0.677 & $<0.001$ & 0.080 \\
\hline$c 9, t 12-$ & 17.1 & 13.4 & 11.0 & 8.69 & 6.86 & 2.33 & $<0.001$ & 0.543 \\
\hline$t 11, c 15-$ & 1.27 & 1.33 & 1.07 & 1.11 & 0.62 & 0.343 & 0.101 & 0.437 \\
\hline$c 9, t 11-$ & 8.02 & 3.83 & 0.26 & 0.57 & 1.39 & 2.178 & 0.022 & 0.092 \\
\hline CLA $t t$ & 12.3 & 12.7 & 10.8 & 11.9 & 14.9 & 3.18 & 0.536 & 0.336 \\
\hline$t 9, c 12-$ & 21.5 & 15.4 & 12.9 & 10.3 & 9.36 & 2.80 & $<0.001$ & 0.047 \\
\hline Sum & 69.0 & 53.0 & 41.4 & 36.8 & 36.5 & 8.83 & $<0.001$ & 0.018 \\
\hline \multicolumn{9}{|l|}{ Oxo FA } \\
\hline 10-охо-18:0 & 147 & 180 & 175 & 163 & 162 & 17.9 & 0.819 & 0.280 \\
\hline 13-охо-18:0 & 24.1 & 30.6 & 30.0 & 30.8 & 31.8 & 5.09 & 0.019 & 0.192 \\
\hline Sum & 171 & 210 & 205 & 194 & 194 & 19.9 & 0.354 & 0.273 \\
\hline Total gain & 1663 & 1613 & 1420 & 1512 & 1572 & 259.4 & 0.544 & 0.364 \\
\hline
\end{tabular}


The BH/VFA ratios, showed in Table 2, were calculated in order to evaluate the relation conversely between $\mathrm{BH}$ and fermentative activity. The $\mathrm{BH} / \mathrm{VFA}$ ratios for $c 9-18: 1$ and $c 9, c 12-18: 2$ increased linearly with CT dose, whereas the BH/VFA ratio for $c 9, c 12, c 15-$ 18:3 was unaffected by CT dose.

Table 3 shows the effect of increasing doses of CT on the balance of C18 FA during the $24 \mathrm{~h}$ of incubation. The $c 9-18: 1 ; c 9, c 12-18: 2$ and $c 9, c 12, c 15-18: 3$, the main dietary unsaturated C18 FAs, displayed large negative balances (losses) in all treatments. The losses of $c 9-18: 1 ; c 9, c 12-18: 2$ and $c 9, c 12, c 15-18: 3$ did not differ among treatments $(p>0.05)$.

The C18 FAs displaying a positive balance (i.e., biohydrogenation products-BHP) comprise the 18:0, 18:1 and 18:2 isomers and oxo-FA. Occasionally, other C18 FAs (i.e., c11-18:1) presented negative balances, but the overall pattern presented positive balances. Stearic acid (18:0) varied from $656 \mu \mathrm{g} /$ tube to $774 \mu \mathrm{g} /$ tube and did not differ significantly among the CT doses tested. All 18:1 isomers, except c11-18:1, displayed consistently positive balances that were larger for $t 11-18: 1$, from $324 \mu \mathrm{g} /$ tube to $456 \mu \mathrm{g} / \mathrm{tube}$, and was not affected by CT. The $t 4-, t 5-, t 6-+t 7-+t 8-, t 15-$ and $t 16+t 14-18: 1$ isomers increased linearly with the increasing CT doses, whereas the t9-18:1 isomer decreased linearly, and none of the other 18:1 isomers identified was significantly affected by C. ladanifer CT extract $(p>0.05)$.

Collectively, the 18:2 isomers changed from $37 \mu \mathrm{g} / \mathrm{tube}$ (in 75 and $100 \mathrm{~g} / \mathrm{kg}$ DM of C. ladanifer CT extract) to $69 \mu \mathrm{g} /$ tube of the total BHP formed (in the control). The 18:2 isomers showed a quadratic response, with highest values in the control $(p=0.018)$ than in CT treatments. The major individual 18:2 isomer was the $t 9, c 12-18: 2$, which ranged from $9.4 \mu \mathrm{g} /$ tube to $21.5 \mu \mathrm{g} /$ tube and presented a quadratic effect $(p=0.047)$ similar to the 18:2 isomers sum. The $c 9, t 11-, t 9, t 12-$ and $c 9, t 12-18: 2$ decreased linearly with increasing CT doses. Relative to oxo-FAs, only the 13-oxo-18:0 increased linearly $(p=0.019)$ with CT dose whereas the others oxo-FAs were unaffected.

Table 4 shows the effect of increasing CT doses on the balance of other non-C18 FAs, mostly derived from microbial de novo synthesis, and DMAs during the $24 \mathrm{~h}$ of incubation. Most of these FAs presented a positive balance, except for 12:0, 20:1 and c7-16:1. The 14:0, 16:0, cyclo-17:0 and 26:0 FA decreased linearly with the CT dose, whereas the 28:0 and c7-16:1 presented a quadratic effect, with lower values for 50 and $75 \mathrm{~g} / \mathrm{kg}$ DM of C. ladanifer CT extract in 28:0, and for $50 \mathrm{~g} / \mathrm{kg}$ DM of C. ladanifer CT extract in c7-16:1.

All the odd- and branched-chain FAs (OBCFAs) were affected by CT and showed a similar trend, decreasing linearly with the CT dose, with exception for the 13:0, which showed a quadratic response, with higher value in the control (without CT supplementation) and lower in $75 \mathrm{~g} / \mathrm{kg}$ DM of C. ladanifer CT extract.

The total of DMAs comprised from 255 to $721 \mu \mathrm{g} / \mathrm{g}$ of DM and presented a quadratic response to the CT dose. All of the DMAs decreased linearly with the CT dose, except for 18:1, which presented a quadratic response, with a lower value in the $75 \mathrm{~g} / \mathrm{kg} \mathrm{DM}$ of $C$. ladanifer CT extract. 
Table 4. Effect of increasing doses of Cistus ladanifer condensed tannin (CT) extract $(0,25,50,75$ and $100 \mathrm{~g} / \mathrm{kg} \mathrm{DM}$ ) on other fatty acids (FAs) and dimethyl acetals (DMAs) balance (ug/g DM). OBCFAs: odd- and branched-chain FAs.

\begin{tabular}{|c|c|c|c|c|c|c|c|c|}
\hline & \multicolumn{5}{|c|}{ C. ladanifer CT, g/kg Dry Matter } & \multirow{2}{*}{ SEM } & \multicolumn{2}{|c|}{$p$ Values } \\
\hline & 0 & 25 & 50 & 75 & 100 & & Linear & Quadratic \\
\hline \multicolumn{9}{|l|}{ FA } \\
\hline $12: 0$ & -99.8 & -96.7 & -146 & -79.7 & -103 & 34.67 & 0.921 & 0.619 \\
\hline $14: 0$ & 190 & 181 & 85.3 & 98.6 & 62.0 & 26.6 & $<0.001$ & 0.591 \\
\hline $16: 0$ & 1456 & 1274 & 316 & 785 & 329 & 347.1 & 0.021 & 0.507 \\
\hline $\begin{array}{c}\text { Cyclo- } \\
17: 0\end{array}$ & 37.0 & 54.9 & 23.2 & 10.4 & -10.2 & 13.10 & 0.003 & 0.252 \\
\hline $20: 0$ & 50.6 & 47.3 & 13.3 & 37.4 & 22.4 & 13.69 & 0.141 & 0.207 \\
\hline $20: 1$ & -7.38 & -6.85 & -18.8 & -10.7 & -11.3 & 4.524 & 0.423 & 0.307 \\
\hline 21:0 & 0.94 & 3.03 & 0.34 & -0.80 & 3.64 & 1.32 & 0.707 & 0.213 \\
\hline $22: 0$ & 43.9 & 37.1 & -3.93 & 21.0 & 14.0 & 15.99 & 0.149 & 0.056 \\
\hline $23: 0$ & 9.74 & 5.80 & 2.30 & 6.50 & 4.99 & 2.708 & 0.316 & 0.229 \\
\hline $24: 0$ & 39.1 & 34.3 & 10.4 & 25.5 & 10.4 & 10.03 & 0.050 & 0.629 \\
\hline $26: 0$ & 164 & 112 & 72.0 & 87.5 & 69.1 & 17.32 & $<0.001$ & 0.073 \\
\hline $28: 0$ & 8.38 & 10.8 & 0.44 & 0.06 & 10.5 & 3.151 & 0.521 & 0.039 \\
\hline c7-16:1 & -1.77 & -1.45 & -11.82 & -5.74 & -5.67 & 4.276 & 0.063 & 0.041 \\
\hline c9-17:1 & 1.51 & -0.80 & -2.24 & 1.69 & -2.36 & 2.006 & 0.417 & 0.805 \\
\hline \multicolumn{9}{|l|}{ OBCFAs } \\
\hline $13: 0$ & 15.8 & 7.50 & 9.09 & 4.30 & 7.48 & 2.093 & 0.005 & 0.036 \\
\hline $\mathrm{i}-14: 0$ & 28.9 & 26.6 & 19.9 & 16.1 & 14.0 & 5.33 & 0.027 & 0.879 \\
\hline $\mathrm{i}-15: 0$ & 64.4 & 45.5 & 24.7 & 21.6 & 9.42 & 5.06 & $<0.001$ & 0.062 \\
\hline$a-15: 0$ & 139 & 124 & 75.1 & 71.2 & 50.7 & 9.13 & $<0.001$ & 0.334 \\
\hline $15: 0$ & 130 & 111 & 58.0 & 61.6 & 43.8 & 13.5 & $<0.001$ & 0.251 \\
\hline $\mathrm{i}-16: 0$ & 44.2 & 47.8 & 32.2 & 26.7 & 20.2 & 5.36 & $<0.001$ & 0.558 \\
\hline $\mathrm{i}-17: 0$ & 46.8 & 35.8 & 22.7 & 30.6 & 18.6 & 5.36 & 0.001 & 0.322 \\
\hline a-17:0 & 72.5 & 58.1 & 38.0 & 42.3 & 20.8 & 9.61 & $<0.001$ & 0.739 \\
\hline $17: 0$ & 73.4 & 52.3 & 31.4 & 33.8 & 23.5 & 10.14 & 0.001 & 0.228 \\
\hline Total & 615 & 508 & 311 & 308 & 209 & 52.4 & $<0.001$ & 0.301 \\
\hline \multicolumn{9}{|l|}{ DMAs } \\
\hline $12: 0$ & 16.2 & 17.2 & 11.1 & 6.46 & 2.93 & 2.774 & $<0.001$ & 0.327 \\
\hline $\mathrm{i}-14: 0$ & 18.5 & 13.3 & 0.63 & 3.00 & -2.44 & 3.587 & $<0.001$ & 0.254 \\
\hline $14: 0$ & 31.0 & 23.3 & 11.3 & 5.47 & -6.31 & 5.258 & $<0.001$ & 0.911 \\
\hline $\mathrm{i}-15: 0$ & 10.2 & 13.1 & 5.94 & 3.58 & -0.41 & 2.528 & $<0.001$ & 0.300 \\
\hline$a-15: 0$ & 528 & 392 & 260 & 183 & 221 & 54.79 & $<0.001$ & 0.051 \\
\hline $15: 0$ & 9.53 & 8.36 & 2.64 & 1.83 & 0.51 & 2.235 & 0.002 & 0.588 \\
\hline $16: 0$ & 68.5 & 55.9 & 40.7 & 37.0 & 18.4 & 6.28 & $<0.001$ & 0.983 \\
\hline $18: 0$ & 9.80 & 4.18 & 4.59 & 3.28 & 3.80 & 1.864 & 0.018 & 0.087 \\
\hline $18: 1$ & 29.3 & 19.3 & 6.46 & 18.4 & 16.8 & 3.45 & 0.020 & 0.003 \\
\hline Total & 721 & 546 & 343 & 262 & 255 & 58.6 & $<0.001$ & 0.041 \\
\hline
\end{tabular}

SEM, standard error of the mean.

\section{Discussion}

The utilization of $\mathrm{CT}$ extracts from various origins to modulate the ruminal $\mathrm{BH}$ as a way to increase the rumen outflow of healthy FA, such as t11-18:1, $c 9, t 11-18: 2$ and dietary PUFA has been the subject of several studies [19,27-30]. Although some results have shown the potential of this strategy to improve the FA profile of ruminant edible fat, overall, the effects of CT extracts on ruminal metabolism including fermentation [31-35] and $\mathrm{BH}[5-7,12,13,19,27,29,30]$ are inconsistent. The diversity the effects of CT extracts on ruminal fermentation and $\mathrm{BH}$ may be due to several factors, such as $\mathrm{CT}$ composition and concentration, basal substrate, and experimental conditions [19,33,34]. In the current study, the increasing doses of $C$. ladanifer CT reduced the VFA production, which is in disagreement with previous in vitro studies where $100 \mathrm{~g} / \mathrm{kg}$ DM of $C$. ladanifer CT extract did not depress ruminal fermentation [7,12]. The effects on ruminal $\mathrm{BH}$ were also inconsistent with previous in vitro studies using $C$. ladanifer $\mathrm{CT}$ extracts, where the incubation of $100 \mathrm{~g} / \mathrm{kg}$ DM of extract increased the $c 9, c 12-18: 2$ and $c 9, c 12, c 15-18: 3 \mathrm{BH}$ and the t11-18:1 production $[7,12]$. Conversely, in the present work, the incubation of a C. ladanifer CT extract at doses between 25 to $100 \mathrm{~g} / \mathrm{kg}$ DM had a limited effect on $\mathrm{BH}$, affecting only $c 9, c 12, c 15-18: 3 \mathrm{BH}$ that decreased and the production of the minor BHP, without a change to t11-18:1 production. Cistus ladanifer CT extract used in the present work was obtained from 
the same C. ladanifer plants and by the same extraction methodology used in Guerreiro et al., [7]. The tested doses were the same or below to the dose used previously $[7,12]$. However, in the present experiment, different in vitro incubation conditions, such as basal substrate and incubation time, were used, which may help to explain the difference in results on fermentation and $\mathrm{BH}$.

The composition of the basal diet is appointed as one of the reasons for the inconsistent effect of plant secondary metabolites, such as tannins on ruminal fermentation and BH $[19,34,35]$. In the present work, the substrate consisted of a high-concentrate diet while in previous works, with C. ladanifer CT extract, $70 \%$ of the substrate was composed of dehydrated Lucerne $[7,12]$. A shift in the microbial composition and activity associated to substrate composition might have led to distinct fermentation and $\mathrm{BH}$ responses to $C$. ladanifer CT extract $[36,37]$. Moreover, multiple interactions that might occur between $C$. ladanifer $\mathrm{CT}$ and other dietary components $[19,30]$ can contribute to the discrepancy among in vitro experiments with $C$. ladanifer $C T$ extracts. In vivo trials with lambs showed that the effect of the C. ladanifer plant (leaves and soft stems) in ruminal BH is dependent on the characteristics of the basal diets, such as concentrate level and composition, and lipid supplementation [28]. An increase in the t11-18:1 and a reduction in 18:0 contents in abomasal digesta was observed when C. ladanifer $(250 \mathrm{~g} / \mathrm{kg} \mathrm{DM})$ was included in a high-forage diet supplemented with vegetable oils rich in PUFA $(60 \mathrm{~g} / \mathrm{kg}$ DM), but not when it was incorporated in a lipid unsupplemented $\operatorname{diet}$ [15]. On the other hand, the incorporation of $C$. ladanifer (50 and $200 \mathrm{~g} / \mathrm{kg} \mathrm{DM}$ ) in a diet containing 1:1 forage-to-concentrate supplemented with $0,40 \mathrm{or} 80 \mathrm{~g} / \mathrm{kg}$ of vegetable oils increased the ruminal production of t10-18:1 instead t11-18:1, which was exacerbated by the simultaneous inclusion of PUFA and C. ladanifer in diets [18]. Moreover, in a diet containing 1:1 forage-to-concentrate supplemented with 60 $\mathrm{g} / \mathrm{kg}$ of vegetable oil in which the cereal was replaced by dehydrated citrus pulp, the $C$. ladanifer $(150 \mathrm{~g} / \mathrm{kg})$ had a minor effect on the fatty acid composition of intramuscular fat [38]. Conversely, when combined with cereals, C. ladanifer led to an increase in the 18:0 and reduced the $\mathrm{BH}$ intermediates in intramuscular fat, suggesting that under these conditions. C. ladanifer increases the ruminal BH completeness [38]. As suggested by the in vivo experiments with the $C$. ladanifer plant, interactions between the basal substrate and C. ladanifer $\mathrm{CT}$ may have occurred, resulting in distinct $\mathrm{CT}$ responses when the composition of the basal diet is changed.

Incubation time should also be considered in the interpretation of the present results. Relative to previous in vitro trials with $C$. ladanifer $\mathrm{CT}$ extracts, in the present work, a longer incubation time was used ( $6 \mathrm{~h}$ vs. $24 \mathrm{~h}$ ). In long incubation times, similar BH endpoints might be achieved independently to incur differences among treatments at earlier stages of the incubation [39]. Unfortunately, the FA profile in ruminal fluid was not monitored during incubation, and the possibility of the $C$. ladanifer $\mathrm{CT}$ response varying over incubation time cannot be excluded.

Odd- and branched-chain FAs (OBCFAs) in rumen are mainly present in membrane lipids of rumen microbes, which are able to synthetize them from propionate and/or branched-chain VFAs derived from branched-chain amino acids, and incorporate them into their cell membranes [40]. Rumen bacterial lipids are also characterized by a high content of plasmalogens, a subclass of phospholipids containing alk-1-enyl (vinyl) ether chains which can be detected by the presence of DMA released under acid catalysis [26]. Thus, OBCFAs and DMAs have been proposed as microbial markers in the rumen ecosystem [41-43]. The general decrease in OBCFA and DMA production with increasing doses of $C$. ladanifer $\mathrm{CT}$ associated with a reduction in VFA production suggests a reduction of bacterial activity. In fact, CT has a inhibitory effect on the activity and growth of microorganisms, which is probably due to their ability to form complexes with various types of molecules such as proteins, polysaccharides and minerals, allowing them to interact with microorganisms' membranes, cell walls and extracellular structures and also reduce nutrient availability [44].

The inhibitory effects of $C$. ladanifer CT were more pronounced for OBCFA and DMA production (up to a $65 \%$ reduction with $100 \mathrm{~g} / \mathrm{kg}$ DM of C. ladanifer CT), than for the 
total VFA production (a $27 \%$ reduction with $100 \mathrm{~g} / \mathrm{kg}$ DM of C. ladanifer CT). Moreover, the BH of $c 9-18: 1$ and $c 9, c 12-18: 2$ was not affected by increasing doses of $C$. ladanifer $C T$, although the BH of $c 9, c 12, c 15-18: 3$ was depressed. The basal substrate was supplemented with $60 \mathrm{~g} / \mathrm{kg}$ of sunflower oil, which contains $c 9, c 12-18: 2$ as the main FA, followed by $c 9-18: 1$, while $c 9, c 12, c 15-18: 3$ is absent from sunflower oil and thus it was provided in small amounts from feed structural lipids. Thus, the availability of $c 9, c 12, c 15-18: 3$ and its subsequent $\mathrm{BH}$ was dependent on feed material degradation, and closely followed the VFA production, as it is evidenced by constant ratio between $c 9, c 12, c 15-18: 3 \mathrm{BH}$ and VFA production.

Thus, it is apparent that there was an uncoupling between $c 9-18: 1$ and $c 9, c 12-18: 2 \mathrm{BH}$ activity and other microbial metabolic activities, but within the other microbial metabolic activities, and also between the ATP energy yielding catabolism (i.e., VFA production) and microbial growth (i.e., OBCFA and DMA production). The ATP yield is expected to be closely related to the VFA produced, as VFAs are end-products of the fermentative pathways [45]. The ATP obtained is expected to support the microbial growth. The uncoupling between energy yield and growth means that energy is being produced (i.e., VFAs) but not translated in obvious growth (i.e., evaluated by the yield in microbial markers such as BCFAs and DMAs). Polyunsaturated fatty acids (PUFAs) have well established bacteriostatic effects and it is reported that, in pure cultures studies, Butyrivibrio fibrisolvens growth was initiated only when PUFAs $(c 9, c 1218: 2$ and $c 9, c 12, c 15-18: 3)$ had been metabolized and reduced to t11-18:1 [46]. The presence of a high concentration of CT can also exert disruptive effects on microbial cell membranes [44,47]. Some bacteria are able to handle toxic environmental stimuli by increasing the trans-18:1 esterified in cell membrane phospholipids and thus diminishing its fluidity [48]. Moreover, trans-18:1 has also been reported as a growth promoter for some bacteria [49]. Thus, rumen $\mathrm{BH}$ might confer protection to rumen microbiota subjected to high PUFA and high CT concentrations by decreasing the PUFA concentrations and simultaneously by increasing the availability of trans-18:1 to be incorporated in rumen microbiota cell membranes [9]. In the present experiment, the substrate incubated contained a high PUFA concentration (i.e., $60 \mathrm{~g} / \mathrm{kg}$ of sunflower oil) and increasing amounts of CT. So, in response to these adverse conditions, the microbial population may have to limit growth and fermentative activity while maintaining the BH pathways in order to reduce the concentration of PUFAs and generate trans-18:1 and incorporate it into their membranes. The maintenance of a higher BH activity, compared to growth and fermentative activity, in response to increasing doses of CT is consistent with the hypothesis that $\mathrm{BH}$ is an adaptive stress response against not only a high PUFA concentration but also against other membrane disruptors, such as CT. The disruptive effects of CTs on the cell membrane might also explain the apparent uncoupling between ATP production and growth observed in the present experiment, as increased energy would be used for the maintenance of ion gradients across the damaged cell membrane [50].

Condensed tannins have been reported to inhibit the last step of $\mathrm{BH}$, decreasing the 18:0 formation and leading to $t 11-18: 1$ accumulation $[5,6,11]$. In the present study, no effect of C. ladanifer CT on 18:0 and on 18:1 isomers yield was observed, which confirms previous observations with the same CT source $[7,12,13]$. In fact, increasing doses of $C$. ladanifer CT did not modify the major BHP, despite the small but significant increase in oxo-FA and decrease in 18:2 BHP. The 10-oxo-18:0 and 13-oxo-18:0 have been described as end products of the $\mathrm{BH}$ of the $c 9-18: 1$ and $c 9, c 12-18: 2$, respectively [26,51,52]. In the present experiment, oxo-FA represented up to $15.4 \%$ of the BHP, which is consistent with previous reports $[7,12,13]$.

\section{Conclusions}

The incubation of increasing doses of C. ladanifer CT extract $(0,25,50,75$ and $100 \mathrm{mg} / \mathrm{g}$ $\mathrm{kg}$ DM) using oil-supplemented high-concentrate subtract led to a moderate decrease in VFA production and to a very pronounced depression of OBCFA and DMA production, without affecting the $\mathrm{BH}$ or the $\mathrm{BH}$ products yield. The apparent uncoupling between 
growth, ATP production and $\mathrm{BH}$ activity could be the reflex of the response of the microbial population to stress induced by high $\mathrm{CT}$ and PUFA concentration in the rumen. In addition to assessing the impact of increasing doses of CT extract from C. ladanifer on ruminal fermentation and $\mathrm{BH}$, it was also our objective determine the C. ladanifer CT extract doses which optimize the $c 9, t 11-18: 2$ and $t 11-18: 1$ production. However, in this in vitro experiment, it was not possible achieve this goal. The present results emphasize the inconsistency regarding the effects of $\mathrm{CT}$ on rumen fermentation and $\mathrm{BH}$. Further studies are needed to elucidate the impact of CT on the ruminal population and metabolism for the adequate use of CT extract as a feed additive to improve the nutritional value of ruminant fat.

Supplementary Materials: The following are available online at https:/ / www.mdpi.com/2076-261 5/11/3/761/s1, Table S1: Initial ( $0 \mathrm{~h})$ and final ( $24 \mathrm{~h})$ concentration of total volatile fatty acids (VFA, $\mathrm{mmol} / \mathrm{L})$, molar percentages of individual VFA ( $\mathrm{mol} / 100 \mathrm{~mol})$ and $\mathrm{pH}$ during the in vitro rumen incubation with $0,25,50,75$ and $100 \mathrm{~g} / \mathrm{kg}$ DM of Cistus ladanifer condensed tannins extract, Table S2: Initial $(0 \mathrm{~h})$ and final $(24 \mathrm{~h})$ concentration of $\mathrm{C} 18$ fatty acids (FA, $\mu \mathrm{g}$ per tube) during the in vitro rumen incubation with $0,25,50,75$ and $100 \mathrm{~g} / \mathrm{kg}$ DM of Cistus ladanifer condensed tannins extract.

Author Contributions: Conceptualization, O.G., S.P.A., E.J. and R.J.B.B.; methodology, O.G., S.P.A. and M.C.; data curation and statistical analysis O.G.; writing-original draft preparation, O.G.; writing-review and editing, O.G., S.P.A., M.F.D., E.J. and R.J.B.B.; project administration, E.J. and R.J.B.B.; funding acquisition, E.J. and R.J.B.B. All authors have read and agreed to the published version of the manuscript.

Funding: This research was funded by the Fundação para a Ciência e Tecnologia (FCT) through research grants to O.G. (SFRH/BD/84406/2012) and M.C. (SFRH/BD/90468/2012), research contract to S.P.A. (DL 57/2016/CP1438/CT0008) and the projects PTDC/CVT/120122/2012, UIDB/00276/2020 from Centre for Interdisciplinary Research in Animal Health, Faculty of Veterinary Medicine, University of Lisbon and UIDB/05183/2020 from MED-Mediterranean Institute for Agriculture, Environment and Development and by the Alentejo2020 program for financial support to the project "CistusRumen-Sustainable use of Rockrose (Cistus ladanifer L.) in small ruminants-Increase of the competitiveness and reduction of the environmental impact" (ALT20-03-0145-FEDER-000023) co-funded by Regional Development European Foundation.

Institutional Review Board Statement: The study was approved by the Ethical and Animal WellBeing Commission (CEBEA) of the Faculty of Veterinary Medicine, University of Lisbon, Portugal (Protocol FMV/CEBEA 007/2016). All methods and procedures were performed following the established guidelines from this institution and following compliance guidelines of European Union (Directive 86/609/EEC).

Data Availability Statement: Data sharing not applicable.

Conflicts of Interest: The authors declare no conflict of interest.

\section{References}

1. Bhat, T.K.; Singh, B.; Sharma, O.P. Microbial degradation of tannins-A current perspective. Biodegradation 1998, 9, $343-357$.

2. Patra, A.K.; Saxena, J. Exploitation of dietary tannins to improve rumen metabolism and ruminant nutrition. J. Sci. Food Agric. 2011, 91, 24-37.

3. McMahon, L.R.; McAllister, T.A.; Berg, B.P.; Majak, W.; Acharya, S.N.; Popp, J.D.; Coulman, B.E.; Wang, Y.; Cheng, K.J. A review of the effects of forage condensed tannins on ruminal fermentation and bloat in grazing cattle. Can. J. Plant. Sci. 2000, 80, 469-485.

4. McSweeney, C.S.; Palmer, B.; McNeill, D.M.; Krause, D.O. Microbial interactions with tannins: Nutritional consequences for ruminants. Anim. Feed. Sci. Technol. 2001, 91, 83-93.

5. Vasta, V.; Makkar, H.P.S.; Mele, M.; Priolo, A. Ruminal biohydrogenation as affected by tannins in vitro. Br. J. Nutr. 2009, 102, 82-92.

6. Khiaosa-Ard, R.; Bryner, S.F.; Scheeder, M.R.L.; Wettstein, H.R.; Leiber, F.; Kreuzer, M.; Soliva, C.R. Evidence for the inhibition of the terminal step of ruminal $\alpha$-linolenic acid biohydrogenation by condensed tannins. J. Dairy. Sci. 2009, 92, 177-188.

7. Guerreiro, O.; Alves, S.P.; Costa, M.; Cabo, A.; Duarte, M.F.; Jerónimo, E.; Bessa, R.J.B. Effects of extracts obtained from Cistus ladanifer L. on in vitro rumen biohydrogenation. Anim. Feed. Sci. Technol. 2016, 219, 304-312.

8. Vahmani, P.; Ponnampalam, E.N.; Kraft, J.; Mapiye, C.; Bermingham, E.N.; Watkins, P.J.; Proctor, S.D.; Dugan, M.E.R. Bioactivity and health effects of ruminant meat lipids. Invited Review. Meat. Sci. 2020, 165, 108114. 
9. Bessa, R.J.B.; Santos-Silva, J.; Ribeiro, J.M.R.; Portugal, A.V. Reticulo-rumen biohydrogenation and the enrichment of ruminant edible products with linoleic acid conjugated isomers. Livest. Prod. Sci. 2000, 63, 201-211.

10. Vasta, V.; Bessa, R.J.B. Manipulating ruminal biohydrogenation by the use of plants bioactive compounds. In Dietary Phytochemicals and Microbes; Patra, A.K., Ed.; Springer: Dordrecht, The Netherlands, 2012; pp. 263-284.

11. Buccioni, A.; Minieri, S.; Rapaccini, S.; Antongiovanni, M.; Mele, M. Effect of chestnut and quebracho tannins on fatty acid profile in rumen liquid- and solid-associated bacteria: An in vitro study. Animal 2011, 5, 1521-1530.

12. Costa, M.; Alves, S.P.; Cabo, A.; Guerreiro, O.; Stilwell, G.; Dentinho, M.T.; Bessa, R.J.B. Modulation of in vitro rumen biohydrogenation by Cistus ladanifer tannins compared with other tannin sources. J. Sci. Food Agric. 2017, 97, 629-635.

13. Carreño, D.; Hervás, G.; Toral, P.G.; Belenguer, A.; Frutos, P. Ability of different types and doses of tannin extracts to modulate in vitro ruminal biohydrogenation in sheep. Anim. Feed Sci. Technol. 2015, 202, 42-51.

14. Guerreiro, O.; Dentinho, M.T.; Moreira, O.C.; Guerra, A.R.; Ramos, P.A.B.; Bessa, R.J.B.; Duarte, M.F.; Jerónimo, E. Potential of Cistus ladanifer L. (rockrose) in small ruminant diets-effect of season and plant age on chemical composition, in vitro digestibility and antioxidant activity. Grass Forage Sci. 2016, 71, 437-447.

15. Jerónimo, E.; Alves, S.P.; Dentinho, M.T.P.; Martins, S.V.; Prates, J.A.M.; Vasta, V.; Santos-Silva, J.; Bessa, R.J.B. Effect of grape seed extract, Cistus ladanifer L. and vegetable oil supplementation on fatty acid composition of abomasal digesta and intramuscular fat of lambs. J. Agric. Food Chem. 2010, 58, 10710-10721.

16. Jerónimo, E.; Alfaia, C.M.M.; Alves, S.P.; Dentinho, M.T.P.; Prates, J.A.M.; Vasta, V.; Santos-Silva, J.; Bessa, R.J.B. Effect of dietary grape seed extract and Cistus ladanifer L. in combination with vegetable oil supplementation on lamb meat quality. Meat Sci. 2012, 92, 841-847.

17. Francisco, A.; Dentinho, M.T.; Alves, S.P.; Portugal, P.V.; Fernandes, F.; Sengo, S.; Jerónimo, E.; Oliveira, M.A.; Costa, P.; Sequeira, A.; et al. Growth performance, carcass and meat quality of lambs supplemented with increasing levels of a tanniferous bush (Cistus ladanifer L.) and vegetable oils. Meat Sci. 2015, 100, 275-282.

18. Alves, S.P.; Francisco, A.; Costa, M.; Santos-Silva, J.; Bessa, R.J.B. Biohydrogenation patterns in digestive contents and plasma of lambs fed increasing levels of a tanniferous bush (Cistus ladanifer L.) and vegetable oils. Anim. Feed Sci. Technol. 2017, 225, 157-172.

19. Frutos, P.; Hervás, G.; Natalello, A.; Luciano, G.; Fondevila, M.; Priolo, A.; Toral, P.G. Ability of tannins to modulate ruminal lipid metabolism and milk and meat fatty acid profiles. Anim. Feed Sci. Technol. 2020, 269, 114623.

20. Goering, H.K.; Van Soest, P.J. Forage fiber Analyses (Apparatus, Reagents, Procedures, and Some Applications); Agricultural Research Service, USDA: Washington, DC, USA, 1970; p. 20.

21. Santos-Silva, J.; Francisco, A.; Alves, S.P.; Portugal, P.; Dentinho, T.; Almeida, J.; Soldado, D.; Jerónimo, E.; Bessa, R.J.B. Effect of dietary neutral detergent fibre source on lambs growth, meat quality and biohydrogenation intermediates. Meat Sci. 2019, 147, 28-36.

22. Francisco, A.E.; Janíček, M.; Dentinho, T.; Portugal, A.P.V.; Almeida, J.M.; Alves, S.P.; Fialho, L.; Jerónimo, E.; Bessa, R.J.B.; Santos-Silva, J. Effects of alfalfa particle size and starch content in diets on feeding behaviour, intake, rumen parameters, animal performance and meat quality of growing lambs. Meat Sci. 2020, 161, 107964.

23. Bessa, R.J.B.; Alves, S.P.; Jeronimo, E.; Alfaia, C.M.; Prates, J.A.M.; Santos-Silva, J. Effect of lipid supplements on ruminal biohydrogenation intermediates and muscle fatty acids in lambs. European J. Lipid Sci. Technol. 2007, 109, 868-878.

24. Oliveira, M.A.; Alves, S.P.; Santos-Silva, J.; Bessa, R.J.B. Effects of clays used as oil adsorbents in lamb diets on fatty acid composition of abomasal digesta and meat. Anim. Feed Sci. Technol. 2016, 213, 64-73.

25. Jenkins, T.C. Technical note: Common analytical errors yielding inaccurate results during analysis of fatty acids in feed and digesta samples. J. Dairy Sci. 2010, 93, 1170-1174.

26. Alves, S.P.; Santos-Silva, J.; Cabrita, A.R.J.; Fonseca, A.J.M.; Bessa, R.J.B. Detailed dimethylacetal and fatty acid composition of rumen content from lambs fed lucerne or concentrate supplemented with soybean oil. PLoS ONE 2013, 8, e58386.

27. Jerónimo, E.; Pinheiro, C.; Lamy, E.; Dentinho, M.T.; Sales-Baptista, E.; Lopes, O.; Silva, F. Tannins in ruminant nutrition: Impact on animal performance and quality of edible products. In Tannins: Biochemistry, Food Sources and Nutritional Properties; Combs, C.A., Ed.; Nova Science Publisher Inc.: Hauppauge, NY, USA, 2016; pp. 121-168.

28. Jerónimo, E.; Dentinho, M.T.; Guerreiro, O.; Francisco, A.; Soldado, D.; Alves, S.P.; Santos-Silva, J.; Bessa, R.J.B. Cistus ladanifer L. in Ruminant Diets-A Sustainable Approach to Improve the Feed Nutritional Value and the Quality of Edible Products. In Advances in Animal Health, Medicine and Production; Springer: Berlin/Heidelberg, Germany, 2020; pp. 128-160.

29. Purba, R.A.P.; Paengkoum, P.; Paengkoum, S. The links between supplementary tannin levels and conjugated linoleic acid (CLA) formation in ruminants: A systematic review and meta-analysis. PLoS ONE 2020, 15, e0216187.

30. Vasta, V.; Daghio, M.; Cappucci, A.; Buccioni, A.; Serra, A.; Viti, C.; Mele, M. Invited review: Plant polyphenols and rumen microbiota responsible for fatty acid biohydrogenation, fiber digestion, and methane emission: Experimental evidence and methodological approaches. J. Dairy Sci. 2019, 102, 3781-3804.

31. Frutos, P.; Hervas, G.; Giraldez, F.J.; Mantecon, A.R. An in vitro study on the ability of polyethylene glycol to inhibit the effect of quebracho tannins and tannic acid on rumen fermentation in sheep, goats, cows, and deer. Aust. J. Agr. Res. 2004, 55, 1125-1132.

32. Hatew, B.; Stringano, E.; Mueller-Harvey, I.; Hendriks, W.H.; Carbonero, C.H.; Smith, L.M.J.; Pellikaan, W.F. Impact of variation in structure of condensed tannins from sainfoin (Onobrychis viciifolia) on in vitro ruminal methane production and fermentation characteristics. J. Anim. Physiol. Nutr. 2016, 100, 348-360. 
33. Jayanegara, A.; Kreuzer, M.; Leiber, F. Ruminal disappearance of polyunsaturated fatty acids and appearance of biohydrogenation products when incubating linseed oil with alpine forage plant species in vitro. Livestock Sci. 2012, 147, $104-112$.

34. Bodas, R.; Prieto, N.; Garcia-Gonzalez, R.; Andres, S.; Giraldez, F.J.; Lopez, S. Manipulation of rumen fermentation and methane production with plant secondary metabolites. Anim. Feed Sci. Technol. 2012, 176, 78-93.

35. Klevenhusen, F.; Muro-Reyes, A.; Khiaosa-Ard, R.; Metzler-Zebeli, B.U.; Zebeli, Q. A meta-analysis of effects of chemical composition of incubated diet and bioactive compounds on in vitro ruminal fermentation. Anim. Feed Sci. Technol. 2012, 176, 61-69.

36. Belanche, A.; Doreau, M.; Edwards, J.E.; Moorby, J.M.; Pinloche, E.; Newbold, C.J. Shifts in the rumen microbiota due to the type of carbohydrate and level of protein ingested by dairy cattle are associated with changes in rumen fermentation. J. Nutr. 2012, 142, 1684-1692.

37. Snelling, T.J.; Auffret, M.D.; Duthie, C.-A.; Stewart, R.D.; Watson, M.; Dewhurst, R.J.; Roehe, R.; Walker, A.W. Temporal stability of the rumen microbiota in beef cattle, and response to diet and supplements. Animal Microbiome 2019, 1, 1-14.

38. Francisco, A.; Alves, S.P.; Portugal, P.V.; Dentinho, M.T.; Jerónimo, E.; Sengo, S.; Almeida, J.; Bressan, M.C.; Pires, V.M.R.; Alfaia, C.M.; et al. Effects of dietary inclusion of citrus pulp and rockrose soft stems and leaves on lamb meat quality and fatty acid composition. Animal 2018, 12, 872-881.

39. Enjalbert, F.; Eynard, P.; Nicot, M.C.; Troegeler-Meynadier, A.; Bayourthe, C.; Moncoulon, R. In vitro versus in situ ruminal biohydrogenation of unsaturated fatty acids from a raw or extruded mixture of ground canola seed/canola meal. J. Dairy Sci. 2003, 86, 351-359.

40. Kaneda, T. Iso-fatty and anteiso-fatty acids in bacteria-Biosynthesis, function, and taxonomic significance. Microbiol. Rev. 1991, 55, 288-302.

41. Bessa, R.J.B.; Maia, M.R.G.; Jerónimo, E.; Belo, A.T.; Cabrita, A.R.J.; Dewhurst, R.J.; Fonseca, A.J.M. Using microbial fatty acids to improve understanding of the contribution of solid associated bacteria to microbial mass in the rumen. Anim. Feed Sci. Technol. 2009, 150, 197-206.

42. Vlaeminck, B.; Fievez, V.; Cabrita, A.R.J.; Fonseca, A.J.M.; Dewhurst, R.J. Factors affecting odd- and branched-chain fatty acids in milk: A review. Anim. Feed Sci. Technol. 2006, 131, 389-417.

43. Costa, M.; Alves, S.; Cappucci, A.; Cook, S.R.; Duarte, A.; Caldeira, R.; McAllister, T.A.; Bessa, R.J.B. Effects of condensed and hydrolysable tannins on rumen metabolism with emphasis on the biohydrogenation of unsaturated fatty acids. J. Agr. Food Chem. 2018, 66, 3367-3377.

44. Smith, A.H.; Zoetendal, E.; Mackie, R.I. Bacterial mechanisms to overcome inhibitory effects of dietary tannins. Microb. Ecol. 2005, 50, 197-205.

45. Russell, J.B.; Wallace, R.J. Energy-yielding and energy-consuming reactions. In The Rumen Microbial Ecosystem; Springer: Berlin/Heidelberg, Germany, 1997; pp. 246-282.

46. Maia, M.R.G.; Chaudhary, L.C.; Bestwick, C.S.; Richardson, A.J.; McKain, N.; Larson, T.R.; Graham, I.A.; Wallace, R.J. Toxicity of unsaturated fatty acids to the biohydrogenating ruminal bacterium, Butyrivibrio fibrisolvens. BMC Microbiol. 2010, 10, 52.

47. Jones, G.A.; McAllister, T.A.; Muir, A.D.; Cheng, K.J. Effects of sainfoin (Onobrychis viciifolia scop) condensed tannins on growth and proteolysis by 4 strains of ruminal bacteria. Appl. Environ. Microb. 1994, 60, 1374-1378.

48. Eberlein, C.; Baumgarten, T.; Starke, S.; Heipieper, H.J. Immediate response mechanisms of Gram-negative solvent-tolerant bacteria to cope with environmental stress: Cis-trans isomerization of unsaturated fatty acids and outer membrane vesicle secretion. Appl. Microbiol. Biotechnol. 2018, 102, 2583-2593.

49. Endo, Y.; Kamisada, S.; Fujimoto, K.; Saito, T. Trans. fatty acids promote the growth of some Lactobacillus strains. J. Gen. Appl. Microbiol. 2006, 52, 29-35.

50. Hackmann, T.; Firkins, J. Maximizing efficiency of rumen microbial protein production. Front. Microbiol. $2015,6,465$.

51. Jenkins, T.C.; AbuGhazaleh, A.A.; Freeman, S.; Thies, E.J. The production of 10-hydroxystearic and 10-ketostearic acids is an alternative route of oleic acid transformation by the ruminal microbiota in cattle. J. Nutr. 2006, 136, 926-931.

52. McKain, N.; Shingfield, K.J.; Wallace, R.J. Metabolism of conjugated linoleic acids and 18: 1 fatty acids by ruminal bacteria: Products and mechanisms. Microbiol. SGM 2010, 156, 579-588. 